\title{
Optimal Estimation of Homogeneous Vectors
}

\author{
Matthias Mühlich ${ }^{1,2}$ and Rudolf Mester ${ }^{1}$ \\ 1 Computer Vision Group, Goethe University, 60054 Frankfurt, Germany \\ \{muehlich, mester\}@iap.uni-frankfurt.de \\ 2 Lehrstuhl für Bildverarbeitung, RWTH Aachen, \\ Sommerfeldstr. 24, 52074 Aachen, Germany
}

\begin{abstract}
Estimation of inhomogeneous vectors is well-studied in estimation theory. For instance, given covariance matrices of input data allow to compute optimal estimates and characterize their certainty. But a similar statement does not hold for homogeneous vectors and unfortunately, the majority of estimation problems arising in computer vision refers to such homogeneous vectors...

The aim of this paper is twofold: First, we will describe several iterative estimation schemes for homogeneous estimation problems in a unified framework, thus presenting the missing link between those apparently different approaches. And secondly, we will present a novel approach called IETLS (for iterative equilibrated total least squares) which is insensitive to data preprocessing and shows better stability in presence of higher noise levels where other schemes often fail to converge.
\end{abstract}

\section{Introduction: The Homogeneous Estimation Problem}

Parameter estimation problems of the general form

$$
\varphi\left(\overline{\mathbf{x}}_{i}, \mathbf{p}\right)=0 \quad \forall \quad i=1, \ldots, m
$$

are ubiquitous in computer vision. Here $\mathbf{p}$ stands for the parameter vector that has to be estimated and $\overline{\mathbf{x}}_{i} \in \mathbb{R}^{\ell}$ denotes some true but unknown vectors (bar accent for true values; index $i=1, \ldots, m$ for different measurements), of which only some error-prone versions

$$
\mathbf{x}_{i}=\overline{\mathbf{x}}_{i}+\mathbf{e}_{i}
$$

(additive noise model) are available. For instance, $\mathbf{x}_{i} \in \mathbb{R}^{4}$ could be the stacked coordinates of corresponding points in a stereo image. When $\overline{\mathbf{x}}_{i}$ is replaced by $\mathbf{x}_{i}$ in (1), we only achieve approximate equality: $\varphi\left(\mathbf{x}_{i}, \mathbf{p}\right) \approx 0$. In statistical literature, this approach is known as errors-in-variables (EIV) model; equations (1) and (2) define data model and error model, respectively. The estimation problem can now be rephrased as obtaining statistically optimal estimates when some statistical information on the errors is given.

Projection from the 3D world to one or several 2D image(s) cancels out the scale factor - this is the underlying reason why the majority of estimation problems in computer vision refer to homogeneous vectors, i.e. we can only estimate 
the sought parameter vector $\mathbf{p}$ up to scale. By normalizing homogeneous vectors to unit vectors, these estimation problems can be linked to directional statistics; distributions of vectors with undefined sign are also known as axial distributions [1]. Homogeneous estimation therefore means that we are estimating on a unit hyperspheres and iterative schemes which are motivated from real space (e.g. variational approaches) do not consider the curved and ambiguous nature of our estimation space. For instance, classical point data covariance matrices are (at most) defined in a tangent hyperplane (here: additionally after declaring one hemisphere as 'valid'). Obviously, this can only be meaningful if the axial distribution is highly concentrated around a preferred axis. For higher error levels, concepts from real space cannot be transferred easily; this is the root of all problems in homogeneous estimation...

All homogeneous data model constraints (1) can be represented as orthog-

onality constraints $\mathbf{a}_{i}^{T} \mathbf{p} \approx 0$. Stacking different measurements on top of each other then results in a matrix equation $\mathbf{A p} \approx \mathbf{0}$. Such Estimation problems are known as (homogeneous) total least squares (TLS) problems [2]. If we additionally allow ancillary constraints $\psi_{j}(\mathbf{p})=0$ (e.g. a single constraint which enforces zero determinant for fundamental matrix estimation), the TLS problem formulation is the general mathematical model for homogeneous estimation. This paper will examine statistically optimal estimation for such models.

\section{Error Models and Cost Functions}

The additive TLS error model is defined by $\mathbf{A}=\overline{\mathbf{A}}+\mathbf{D}$ with a true data matrix $\overline{\mathbf{A}}$ and an error matrix $\mathbf{D}$, both of which being unknown. In this section, we will answer the question how to exploit information on the error model for a statistically optimized estimate of the sought parameter vector and present existing approaches in a unified framework.

\subsection{TLS-Based Estimation Approaches}

The first and second order statistical moments of the random matrix $\mathbf{D}$ can be used to describe the error model. In the same way as mean vector and covariance matrix describe random vectors, a mean matrix $\mathbf{E}[\mathbf{D}]$ and a covariance tensor $\mathcal{C}_{\mathbf{A}}$ (of tensor $\operatorname{rank} 4 ;\left(\mathcal{C}_{\mathbf{A}}\right)_{i p j q}=\operatorname{Cov}\left[(\mathbf{D})_{i p},(\mathbf{D})_{j q}\right]$ ) characterize the statistical properties of random matrices like $\mathbf{D}$. Without loss of generality, we can assume zero-mean errors (we can always subtract E $[\mathbf{D}]$ from the measured data matrix A if necessary), but the covariance tensor is much more problematic. It is definitely non-trivial to exploit this information in a statistically optimal way.

The TLS solution is widely equated with the right singular vector of $\mathbf{A}$ corresponding to the smallest singular value. This narrows the view on the potential of TLS-based approaches considerably because it minimizes the squared algebraic distance $|\mathbf{A p}|^{2}$ under the constraint $|\mathbf{p}|^{2}=1$, i.e. the cost function 


$$
J_{\mathrm{AD}}=\frac{\mathbf{p}^{T} \mathbf{A}^{T} \mathbf{A} \mathbf{p}}{\mathbf{p}^{T} \mathbf{p}}=\sum_{i=1}^{m} \frac{\mathbf{p}^{T} \mathbf{S}_{i} \mathbf{p}}{\mathbf{p}^{T} \mathbf{p}} \quad \text { with } \quad \mathbf{S}_{i}=\mathbf{a}_{i} \mathbf{a}_{i}^{T}
$$

and with $\mathbf{a}_{i}^{T}$ denoting the $i$-th row vector of $\mathbf{A}$. In section 2.2 , we will prove that minimizing $J_{\mathrm{AD}}$ is statistically optimal if and only if the noise in the elements of the TLS data matrix $\mathbf{A}$ is independent and identically distributed (iid).

It is important to stress that taking the right singular vector is just one variant of TLS-based methods; this method will be denoted plain TLS or PTLS from now on. The iid noise assumption connected with PTLS is often not very realistic; therefore, PTLS estimates can be very erroneous (e.g. highly biased in case of fundamental matrix estimation without prior data normalization). ${ }^{1}$

\subsection{Cost Functions in Parameter Space}

In the introduction, we presented the TLS model as general model for estimation of homogeneous vectors. PTLS does not consider covariance information at all, but such information can easily be taken into account, at least if we limit the general model: we will assume that we have a set of measurements $\mathbf{x}_{i}$, each of them can have an arbitrary covariance matrix $\mathbf{B}_{i}$, but they are assumed to be uncorrelated. ${ }^{2}$ Then a minimum mean squared error (MMSE) estimate can be found by minimizing the cost function

$$
J_{\mathrm{ML}}=\sum_{i=1}^{m}\left(\hat{\mathbf{x}}_{i}-\mathbf{x}_{i}\right)^{T} \mathbf{B}_{i}^{-1}\left(\hat{\mathbf{x}}_{i}-\mathbf{x}_{i}\right)
$$

with $\hat{\mathbf{x}}_{i}$ being the estimates for true values of the measurements, i.e. estimates which exactly fulfill the data model (throughout this paper, quantities with a hat symbol on top will always denote estimated values). For Gaussian noise, this is also a maximum likelihood estimate (hence the ML subscript).

Unfortunately, the TLS data matrix $\mathbf{A}$ is in general non-linear in the measurements $\mathbf{x}_{i}$. This makes minimization of $J_{\mathrm{ML}}$ intractable and therefore several different schemes were proposed which effectively replace (4) by an iterative solution of simple minimization problems. The most important existing methods of this class are Renormalization [6], Heteroscedastic Errors-In-Variables (HEIV, [7]), and Fundamental Numerical Scheme (FNS, [8]). All these methods are first

\footnotetext{
${ }^{1}$ Note that the iid noise assumption in the TLS data matrix $\mathbf{A}$ is (in general) even violated if the underlying measurements $\mathbf{x}_{i}$ contain iid noise because of the nonlinearity of the constraints in (1) with respect to the measurements (e.g. conic fitting: linear in the six homogenous conic parameters, quadratic in the measurements). This effect is known as heteroscedasticity [3]. A preprocessing of the data can be used to alleviate the negative effects of heteroscedasticity; this technique is known as conditioning in photogrammetry or data normalization [4] in computer vision. Data normalization works because it makes the errors in $\mathbf{D}$ more iid-like.

2 The full theory including correlated measurements is much more complicated and can be found in the Ph.D. thesis of the first author [5].
} 
order approximations to minimizing $J_{\mathrm{ML}}$; second order errors occur due to the linearization used for covariance propagation from measurements $\mathbf{x}_{i}$ to the TLS data matrix A.

An approximate maximum likelihood cost function $J_{\mathrm{AML}}$ for uncorrelated measurements can be defined as

$$
J_{\mathrm{AML}}=\sum_{i} \frac{\mathbf{p}^{T} \mathbf{S}_{i} \mathbf{p}}{\mathbf{p}^{T} \mathbf{C}_{i} \mathbf{p}} \quad \text { with } \quad \mathbf{S}_{i}=\mathbf{a}_{i} \mathbf{a}_{i}^{T} \quad \text { and } \quad \mathbf{C}_{i}=\operatorname{Cov}\left[\mathbf{a}_{i}\right]
$$

For $\mathbf{C}_{i}=c \mathbf{I}$ with some constant $c$ (iid errors), we find that the algebraic distance $J_{\mathrm{AD}}$ is statistically optimal. A derivation of $J_{\mathrm{AML}}$ can be found in [8], but a much easier one is found in the appendix of [9]; we will summarize the latter one here. Let $r_{i}=\mathbf{a}_{i}^{T} \mathbf{p}$ be the residual (deviation from data model) for the $i$-th measurement and let $\mathbf{r}=\left(r_{1}, \ldots, r_{M}\right)^{T}$ be the vector of residuals. For uncorrelated measurements, the covariance between different residuals is zero and for the variance $\sigma_{i}^{2}$ of the residual $r_{i}$, we find

$$
\sigma_{i}^{2}=\left(\frac{\partial\left(\mathbf{p}^{T} \mathbf{a}_{i}\right)}{\partial \mathbf{x}_{i}}\right) \operatorname{Cov}\left[\mathbf{x}_{i}\right]\left(\frac{\partial\left(\mathbf{a}_{i}^{T} \mathbf{p}\right)}{\partial \mathbf{x}_{i}}\right)^{T}=\mathbf{p}^{T} \mathbf{C}_{i} \mathbf{p}
$$

Thus, the covariance matrix of the residual vector is given by $\boldsymbol{\Sigma}_{\mathbf{r r}}:=\operatorname{Cov}[\mathbf{r}]=$ $\operatorname{diag}\left(\sigma_{i}^{2}\right)$. Minimizing the Mahalanobis norm of the residual vector now means minimizing

$$
J_{\mathrm{AML}}=\mathbf{r}^{T} \boldsymbol{\Sigma}_{\mathbf{r r}}^{-1} \mathbf{r}=\sum_{i=1}^{m}\left(\mathbf{p}^{T} \mathbf{a}_{i}\right) \sigma_{i}^{-2}\left(\mathbf{a}_{i}^{T} \mathbf{p}\right)=\sum_{i=1}^{m} \frac{\mathbf{p}^{T}\left(\mathbf{a}_{i} \mathbf{a}_{i}^{T}\right) \mathbf{p}}{\mathbf{p}^{T} \mathbf{C}_{i} \mathbf{p}}=\sum_{i=1}^{m} \frac{\mathbf{p}^{T} \mathbf{S}_{i} \mathbf{p}}{\mathbf{p}^{T} \mathbf{C}_{i} \mathbf{p}}
$$

which proves (5). We stress that all approaches which do not minimize $J_{\mathrm{ML}}$ are not optimal in the strict sense. However, the degree of approximation errors varies considerably: PTLS gives a coarse and biased estimation, while $J_{\mathrm{AML}}$ defines a criterion whose only difference to the optimal one is a second order error in covariance propagation. ${ }^{3}$

\subsection{A General Framework for All Iterative Approaches}

Different iterative algorithms are presented in totally different form and with very different reasoning: minimizing geometric distances, unbiasing of matrices by subtraction, solving variational equations. But they all share the same essential mathematical core which is determined by the type of problem: estimation of a homogeneous vector. All these methods can be summarized under the following framework:

${ }^{3}$ For TLS models which are linear in both parameters and measurements, both criterions become identical. In general, however, $J_{\mathrm{AML}}$ is a first-order approximation to $J_{\mathrm{ML}}$. Therefore, an estimation scheme need not necessarily be based on $J_{\mathrm{AML}}$. One cannot criticize a scheme for not minimizing $J_{\mathrm{AML}}$ in these situations (for instance, FNS or our new approach do, Renormalization does not); there may be other first-order approximations to $J_{\mathrm{ML}}$ which are statistically equivalent. 
- All methods compute eigenvectors

- The cost function $J_{\mathrm{ML}}$ (or an approximation for it like $J_{\mathrm{AML}}$ ) does not allow closed-form solutions; we therefore have to replace it by "something different" (as defined later) of the general form

$$
J=\frac{\mathbf{p}^{T} \mathbf{X} \mathbf{p}}{\mathbf{p}^{T} \mathbf{p}} .
$$

- The matrix $\mathbf{X}$ will depend on some previous parameter estimate $\mathbf{q}$.

- The cost functions $J(\mathbf{p} ; \mathbf{q})$ in $(8)$ define a sequence of cost functions and every iteration step is essentially $\hat{\mathbf{p}}:=\arg \min _{\mathbf{p}} J(\mathbf{p} ; \mathbf{q})$.

The way in which $\mathbf{X}(\mathbf{q})$ is derived can differ widely, but the general concept behind Sampson method, Fundamental Numerical Scheme, Renormalization, or Heteroscedastic EIV (and later: our new scheme IETLS) is always the same:

1. Set $\mathbf{q}$ to some initial value, e.g. to $\mathbf{q}:=\arg \min _{\mathbf{p}} J_{\mathrm{AD}}=\arg \min _{\mathbf{p}} \sum_{i} \mathbf{S}_{i}$.

2. Compute matrix $\mathbf{X}(\mathbf{q})$ using the previous estimate $\mathbf{q}$.

3. Compute eigenvector of $\mathbf{X}$ corresponding to the smallest eigenvalue. Take this as estimate $\hat{\mathbf{p}}$.

4. Compare $\hat{\mathbf{p}}$ and $\mathbf{q}$. Terminate if similar enough; otherwise set $\mathbf{q}:=\hat{\mathbf{p}}$ and continue at step 2 .

Equation (8) is usually formulated as solving some equation $\mathbf{X p}=\mathbf{0}$ ("variational equation", "renormalization equation" etc). But one actually minimizes an algebraic distance again. From our point of view, (8) emphasizes the structural similarity to $J_{\mathrm{AD}}$. Other related papers only speak of a (hopefully converging) sequence of estimates. We stress that, prior to computation of estimates, every algorithm defines a sequence of cost functions. These cost functions $J(\mathbf{p} ; \mathbf{q})$ will converge "under favorable conditions", and in some cases, one can identify their limit (if existent) as $J_{\mathrm{AML}}$ or any other function which can be expressed in algebraic form. The step from $J_{\mathrm{AML}}$ to a general model for $J$ is found by writing

$$
J_{\mathrm{AML}}=\frac{\mathbf{p}^{T} \mathbf{M}(\mathbf{p}) \mathbf{p}}{\mathbf{p}^{T} \mathbf{p}} \quad \text { with } \quad \mathbf{M}(\mathbf{p})=\sum_{i=1}^{m}\left(\frac{\mathbf{p}^{T} \mathbf{p}}{\mathbf{p}^{T} \mathbf{C}_{i} \mathbf{p}} \mathbf{S}_{i}\right) .
$$

The matrix $\mathbf{M}(\mathbf{p})$ depends on the sought parameter vector-which makes an optimal closed-form solution impossible. But the assumption that current and previous estimate do not differ much allows the following iterative approach: replace $\mathbf{M}(\mathbf{p})$ by some other matrix $\mathbf{X}(\mathbf{q})$ which depends on the previous estimate, thus being independent of $\mathbf{p}$. Then a new estimate can be computed as eigenvector corresponding to the smallest eigenvalue. In the following subsection, we will present different approaches for homogeneous estimation; the unifying framework presented here links them together.

While being based on a statistically justified cost function, all existing approaches share a common problem: If they converge, then their limit has some favorable statistical properties. But the iterative schemes are based on little more than heuristics. As a consequence, these schemes can show severe convergence problems if error levels increase. In section 3, we will present a novel iterative scheme which shows much higher stability to noise. 


\subsection{Sampson's Method, FNS, Renormalization, and HEIV}

An early class of approaches is defined by $\mathbf{X}(\mathbf{q}):=\sum_{i} \alpha_{i}^{2} \mathbf{S}_{i}$ such that the weights $\alpha_{i}$ are functions of the previous estimate. This approach is known as iteratively reweighted TLS. For ellipse fitting, the most obvious choice (cf. (9))

$$
\alpha_{i}^{2}(\mathbf{q})=\frac{\mathbf{q}^{T} \mathbf{q}}{\mathbf{q}^{T} \mathbf{C}_{i} \mathbf{q}} \Rightarrow \mathbf{X}(\mathbf{q}):=\sum_{i} \frac{\mathbf{q}^{T} \mathbf{q}}{\mathbf{q}^{T} \mathbf{C}_{i} \mathbf{q}} \mathbf{S}_{i}
$$

was proposed by Sampson [10]; this defines a first order approximation to the geometric distance between data point and estimated ellipse. Similar approaches were proposed for other computer vision problems like fundamental matrix estimation. These approaches are better than PTLS, but the corresponding series of cost function does not converge to $J_{\mathrm{AML}}$ or any other first order approximation to $J_{\mathrm{ML}}$. Consequently, the estimates are still biased.

In contrast to this, the FNS method [8] has the strong statistical justification of minimizing $J_{\mathrm{AML}}$ and we will summarize it next. At the minimum, the gradient of $J_{\mathrm{AML}}$ with respect to the parameter vector must be zero. Computing the derivative and setting it to zero leads to a so-called "variational equation" which essentially means defining

$$
\mathbf{X}(\mathbf{q}):=\sum_{i=1}^{m}\left(\frac{\mathbf{q}^{T} \mathbf{q}}{\mathbf{q}^{T} \mathbf{C}_{i} \mathbf{q}} \mathbf{S}_{i}-\frac{\left(\mathbf{q}^{T} \mathbf{q}\right)\left(\mathbf{q}^{T} \mathbf{S}_{i} \mathbf{q}\right)}{\left(\mathbf{q}^{T} \mathbf{C}_{i} \mathbf{q}\right)^{2}} \mathbf{C}_{i}\right)=\sum_{i=1}^{m} \frac{\mathbf{q}^{T} \mathbf{q}}{\mathbf{q}^{T} \mathbf{C}_{i} \mathbf{q}}\left(\mathbf{S}_{i}-\frac{\mathbf{q}^{T} \mathbf{S}_{i} \mathbf{q}}{\mathbf{q}^{T} \mathbf{C}_{i} \mathbf{q}} \mathbf{C}_{i}\right)
$$

according to the framework described above. The second summand in (11) can be regarded as a correction term which makes up for inserting $\mathbf{q}$ in place of $\mathbf{p}$.

If this algorithm converges, then it converges to the correct solution (up to the second order errors which distinguish $J_{\mathrm{AML}}$ from $J_{\mathrm{ML}}$ ). But unfortunately, experiments show that the initial value must be very close to the optimum and the noise level must be low; otherwise, the algorithm is likely to diverge. Additionally, the algorithm is extremely sensitive to prior data transformation / normalization; this problem-specific property limits the generality of an algorithm. The reason for all these negative effects is simple: each iteration step silently assumes iid errors again; otherwise, eigensystem-based methods are not optimal. Therefore, the iteration steps are not solved in a statistically optimal way. ${ }^{4}$ Our new algorithm will be free of this drawback.

A third approach, the Renormalization scheme defined by Kanatani [6], has another justification: correction matrices $\mathbf{D}_{i}$ are chosen such that

$$
\mathbf{X}(\mathbf{q}):=\sum_{i=1}^{m} \frac{\mathbf{q}^{T} \mathbf{q}}{\mathbf{q}^{T} \mathbf{C}_{i} \mathbf{q}}\left(\mathbf{S}_{i}-\mathbf{D}_{i}\right) \quad \text { with } \quad \mathrm{E}\left[\frac{\mathbf{q}^{T} \mathbf{S}_{i} \mathbf{q}}{\mathbf{q}^{T} \mathbf{C}_{i} \mathbf{q}}\right]=\mathrm{E}\left[\frac{\mathbf{q}^{T} \mathbf{D}_{i} \mathbf{q}}{\mathbf{q}^{T} \mathbf{C}_{i} \mathbf{q}}\right]
$$

holds, this approach is motivated by subtracting the 'bias' in $\mathbf{S}_{i}$. The term 'bias' is put in quotes because one can show that this bias does not affect the eigen-

${ }^{4}$ One evidence is the need to look for the eigenvalue smallest in absolute value in FNS. A positive definite matrix should not have negative eigenvalues. But the difference in the numerator of $\mathbf{X}$ is dangerous for $\mathbf{p} \neq \mathbf{q}$. 
vectors as long as it is proportional to the identity matrix in expectation (then only the eigenvalues are increased). Therefore, $E\left[\mathbf{q}^{T} \mathbf{S}_{i} \mathbf{q}\right] \neq 0$ is not necessarily a bad sign. Nevertheless, the renormalization approach leads to high quality estimation schemes. However, just like FNS we find that the matrix $\mathbf{X}$ is given as a difference of non-negative definite matrices which is not necessarily non-negative definite as well. Higher errors can lead to convergence problems again.

A further approach is the heteroscedastic errors-in-variables (HEIV) model which was introduced by Leedan [11] and refined by Matei and Meer [7]. A recent paper [12] illustrated the link between FNS and HEIV. Consequently, HEIV shows similar properties and problems (for instance, Nestares et al. report convergence problems in [13]).

The mathematical core of all methods presented so far is very similar: iterative estimation of (generalized) eigenvectors. At the end of this section, we stress that eigenvector-based estimation is not very tolerant to anisotropic (i.e. non-iid) errors. If some eigenvalue $\bar{\lambda}$, corresponding to the true solution $\overline{\mathbf{p}}$, is close to another eigenvalue $\tilde{\lambda}$, corresponding to a wrong estimate $\tilde{\mathbf{p}}$, then very small errors can change the order of eigenvalues. As eigenvectors are mutually orthogonal, a small increase in noise can lead to a completely wrong estimate. This holds for every iteration step. Possible divergence is a severe problem and one should not hope for graceful degradation in homogeneous estimation unless isotropic behavior can be guaranteed for each estimation step. ${ }^{5}$ The deeper mathematical understanding of homogeneous estimation presented so far will now allow us to derive a more stable estimation scheme in the following section.

\section{A Novel Iterative Estimation Approach: IETLS}

The $J_{\mathrm{AML}}$ cost function can be rewritten as

$$
J_{\mathrm{AML}}=\sum_{i} \frac{\mathbf{p}^{T} \mathbf{S}_{i} \mathbf{p}}{\mathbf{p}^{T} \mathbf{C}_{i} \mathbf{p}}=\sum_{i} \frac{\tilde{\mathbf{p}}^{T} \tilde{\mathbf{S}}_{i} \tilde{\mathbf{p}}}{\tilde{\mathbf{p}}^{T} \tilde{\mathbf{C}}_{i} \tilde{\mathbf{p}}}
$$

with $\tilde{\mathbf{p}}=\mathbf{W}_{R}^{-T} \mathbf{p}$ and $\tilde{\mathbf{S}}_{i}=\lambda_{i}^{2} \mathbf{W}_{R} \mathbf{S}_{i} \mathbf{W}_{R}^{T}$ and $\tilde{\mathbf{C}}_{i}=\lambda_{i}^{2} \mathbf{W}_{R} \mathbf{C}_{i} \mathbf{W}_{R}^{T}$. Instead of minimizing $J_{\mathrm{AML}}$ directly in the original coordinate system, we have the freedom to choose weights $\lambda_{i}$ for each data vector and a weight matrix $\mathbf{W}_{R}$ in order to transform the problem of minimizing the same $J_{\mathrm{AML}}$ to a more convenient coordinate system. The cost function $J_{\mathrm{AML}}$ is invariant to transformations of the coordinate system (which is exactly what we can expect from a statistically justified cost function: its result must not depend on the choice of coordinates).

The minimizers $\hat{\mathbf{p}}=\arg \min _{\mathbf{p}}\left(J_{\mathrm{AML}}\right)$ (in the original coordinate system) and $\hat{\tilde{\mathbf{p}}}=\arg \min _{\tilde{\mathbf{p}}}\left(J_{\mathrm{AML}}\right)$ (in the transformed system) are defined only up to scale;

${ }^{5}$ This is also the reason why it is so easy to find limiting statements like "if initialized close to the global optimum" or "under favorable condition" or similar thing written between the lines in algorithmic papers for homogeneous estimation problems. 
therefore the back substitution $\hat{\mathbf{p}}=\mathbf{W}_{R}^{T} \hat{\tilde{\mathbf{p}}}$ of the solution in the transformed space is always a solution in the original space. This basic reweighting technique known as equilibration $[14,15]$ can be seen as a tool to make minimizing $J_{\mathrm{AML}}$ more tractable by choosing a different coordinate system.

It is obvious that equilibration changes error metrics but we have not defined yet how to choose these weights optimally (it is well-known that weight matrices influence the result (e.g. [16]), but the really interesting question is how to find optimal weights). We will answer this question now and develop an iterative scheme based on it. First, we rewrite $J_{\mathrm{AML}}$ as $J_{\mathrm{AML}}=\sum_{i} r_{i}^{2}=\sum_{i} r_{i}^{\prime 2} \mu_{i}^{2}$ with

$$
r_{i}^{2}=\frac{\tilde{\mathbf{p}}^{T} \tilde{\mathbf{S}}_{i} \tilde{\mathbf{p}}}{\tilde{\mathbf{p}}^{T} \tilde{\mathbf{C}}_{i} \tilde{\mathbf{p}}} \quad \text { and } \quad r_{i}^{\prime 2}=\frac{\tilde{\mathbf{p}}^{T} \tilde{\mathbf{S}}_{i} \tilde{\mathbf{p}}}{\tilde{\mathbf{p}}^{T} \tilde{\mathbf{p}}} \quad \text { and } \quad \mu_{i}^{2}=\frac{\tilde{\mathbf{p}}^{T} \tilde{\mathbf{p}}}{\tilde{\mathbf{p}}^{T} \tilde{\mathbf{C}}_{i} \tilde{\mathbf{p}}}
$$

Here, we introduced the raw residuals $r_{i}^{\prime}$ that do not consider the $\mu_{i}^{2}$ weights and the true residuals $r_{i}$ which include the data-dependent denominator. The true residuals are invariant to coordinate transformations (see (13)), but the raw residuals are not. From this point of view, the basic idea behind iterative equilibrated TLS (we will denote this as IETLS) can be described as follows: taking the singular vector corresponding to the smallest singular value (i.e. a PTLS solution) finds the global minimum of $J_{\mathrm{IETLS}}=\sum_{i} r_{i}^{\prime 2}$, i.e. for minimizing the sum of squared raw residuals. By iteratively updating the equilibration weights (i.e. warping the space in which the estimation is carried out) we can make all $\mu_{i}^{2}$ converge to 1 easily. Then the raw residuals converge to the true residuals, the IETLS cost function $J_{\mathrm{IETLS}}$ converges to $J_{\mathrm{AML}}$, and a simple PTLS solution in equilibrated space is now a minimizer of $J_{\mathrm{AML}}$.

Comparing the three functions $J_{\mathrm{AD}}, J_{\mathrm{IETLS}}$, and $J_{\mathrm{AML}}$ shows the difference:

$$
J_{\mathrm{AD}}=\sum_{i} \frac{\mathbf{p}^{T} \mathbf{S}_{i} \mathbf{p}}{\mathbf{p}^{T} \mathbf{p}}, \quad J_{\mathrm{IETLS}}=\sum_{i} \frac{\tilde{\mathbf{p}}^{T} \tilde{\mathbf{S}}_{i} \tilde{\mathbf{p}}}{\tilde{\mathbf{p}}^{T} \tilde{\mathbf{p}}}, \quad J_{\mathrm{AML}}=\sum_{i} \frac{\mathbf{p}^{T} \mathbf{S}_{i} \mathbf{p}}{\mathbf{p}^{T} \mathbf{C}_{i} \mathbf{p}} .
$$

$J_{\mathrm{AD}}$ neglects covariances completely, $J_{\mathrm{AML}}$ is hardly tractable, but $J_{\mathrm{IETLS}}$ includes covariances indirectly by appropriate transformation of coordinates while sharing the same mathematical form as $J_{\mathrm{AD}}$. It is the formerly missing link between the available methods (i.e. computing singular vectors) and the statistically justified cost function $J_{\mathrm{AML}}$. This idea can be used to define an iterative scheme: we first generate initial weights using

$$
\lambda_{i}=\sqrt{\frac{1}{\operatorname{tr}\left[\mathbf{C}_{i}\right]}} \text { and } \mathbf{W}_{R}=\text { itChol }\left[\sum_{i} \lambda_{i}^{2} \mathbf{C}_{i}\right]
$$

where itChol $[\cdot]$ stands for inverting and transposing the Cholesky factor of some matrix. Then define the Basic Iterative Equilibrated TLS Scheme (B-IETLS) as follows:

1. Estimate new parameter $\hat{\mathbf{p}}$ vector in equilibrated space using

$$
\hat{\mathbf{p}}=\mathbf{W}_{R}^{T}\left(\arg \min _{\tilde{\mathbf{p}}} \frac{\tilde{\mathbf{p}} \tilde{\mathbf{S}} \tilde{\mathbf{p}}}{\tilde{\mathbf{p}}^{T} \tilde{\mathbf{p}}}\right) \quad \text { with } \quad \tilde{\mathbf{S}}=\sum_{i} \lambda_{i}^{2} \mathbf{W}_{R} \mathbf{S}_{i} \mathbf{W}_{R}^{T} .
$$


2. Compute new equilibration transformations from

$$
\lambda_{i}=\sqrt{\frac{1}{\mathbf{q}^{T} \mathbf{C}_{i} \mathbf{q}}} \text { and } \quad \mathbf{W}_{R}:=\text { itChol }\left[\sum_{i} \lambda_{i}^{2} r_{i}^{2} \mathbf{C}_{i}\right] .
$$

3. Terminate if estimated $\hat{\mathbf{p}}$ did not change much. Otherwise: next iteration.

It is important to stress that IETLS automatically chooses an appropriate coordinate system in every step. Other iterative schemes implicitly assume that some data normalization (which is a subset of possible right equilibrations) is carried out as a preprocessing step; the correct type of data transformation has to be known in advance and this transformation is not adaptive during the iterative process. The scheme described above is called basic IETLS because it can be refined further. We can decrease computational complexity and simultaneously increase numerical stability (e.g. by computing singular vectors instead of eigenvectors of matrix products) - unfortunately at the expense of longer and less intuitive code. The refined version (including MATLAB sources) can be found in [5], but the general idea should also be clear from B-IETLS.

\section{Experimental Evaluation and Summary}

We successfully applied the IETLS scheme to several computer vision applications including fundamental matrix estimation, homography estimation and orientation estimation (which includes motion estimation as a special case for space-time volumes); see [5] for details and examples. In this general and more theoretical paper, however, we will show numerical simulations.

Figs. 1 shows the mean squared axial error for a simulation in which a homogeneous vector in $\mathbb{P}^{5}$ was estimated (details can be found in [5]). In the left
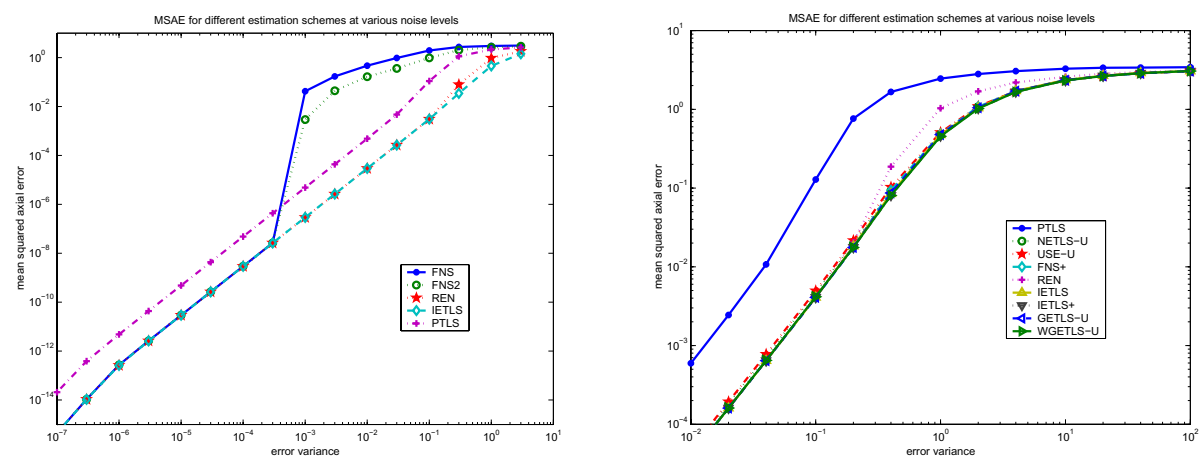

Fig. 1. Mean squared axial error for different noise levels; complete noise range in left image ( $\rightarrow$ FNS often fails to converge) and medium to higher noise levels only in right image $(\rightarrow$ REN shows weaknesses at higher noise levels) 
image, it is clearly visible that FNS breaks down for medium error levels; its estimates even become worse than PTLS. The right image shows a zoom on the upper right part of the curve: renormalization (REN) is more stable but shows slight weaknesses for higher noise levels. In all simulations, IETLS and some variants of it which were also tested perform best.

Summarizing this paper, we emphasize that iterative estimation of homogeneous vectors can be highly sensitive to noise. Therefore, it is not sufficient to define an iterative scheme which converges to some statistically optimized solution-provided that it converges at all. We showed that the essential mathematical core of all homogeneous estimation approaches is some eigensystem analysis. This allowed to identify some underlying general framework and in this framework, we could derive a novel scheme in which each single estimation step is carried out in a statistically optimal way. Experimental evaluation (in this paper and extended evaluation including several computer vision examples in [5]) showed the superiority of our novel approach.

\section{References}

1. Mardia, K.V., Jupp, P.E.: Directional Statistics. Wiley (2000)

2. van Huffel, S., Vandewalle, J.: The Total Least Squares problem: Computational aspects and analysis. SIAM, Philadelphia (1991)

3. Mardia, K.V., Kent, J., Bibby, J.: Multivariate Analysis. Academic Press (1992)

4. Hartley, R.I., Zisserman, A.: Multiple View Geometry in Computer Vision. First edn. Cambridge University Press (2000)

5. Mühlich, M.: Estimation of Homogeneous Vectors and Applications in Computer Vision. PhD thesis, J. W. Goethe-Universität Frankfurt (2004)

6. Kanatani, K.: Statistical Optimization for Geometric Computation: Theory and Practice. Elsevier (1996)

7. Matei, B., Meer, P.: A general method for errors-in-variables problems in computer vision. In: IEEE Conf. CVPR. (2000) 18-25

8. Chojnacki, W., Brooks, M.J., van den Hengel, A., Gawley, D.: On the fitting of surfaces to data with covariances. IEEE Trans. PAMI 22 (2000) 1294-1303

9. Förstner, W.: On Estimating 2D Points and Lines from 2D Points and Lines. In: Festschrift anläßlich des 60. Geburtstages von Prof. Dr.-Ing. Bernhard Wrobel. Technische Universität Darmstadt (2001) $69-87$

10. Sampson, P.D.: Fitting conic sections to 'very scattered' data: An iterative refinement of the Bookstein algorithm. Computer Vision Graphics and Image Processing 18 (1982) 97-108

11. Leedan, Y., Meer, P.: Heteroscedastic regression in computer vision: problems with bilinear constraint. Int. J. Computer Vision 37 (2000) 127-150

12. Chojnacki, W., Brooks, M., van den Hengel, A., Gawley, D.: From FNS to HEIV: A link between two vision parameter estimation methods. IEEE Trans. PAMI 26 (2004) 85-91

13. Nestares, O., Fleet, D.J.: Error-in-variables likelihood functions for motion estimation. In: IEEE Intenational Conference on Image Processing, Barcelona. (2003) 
14. Mühlich, M., Mester, R.: The role of total least squares in motion analysis. In: Proc. Europ. Conf. Comp. Vision. (1998)

15. Mühlich, M., Mester, R.: Subspace methods and equilibration in computer vision. Technical Report XP-TR-C-21, J.W.G.University Frankfurt (1999)

16. Golub, G.H., van Loan, C.F.: Matrix Computations. 2nd edition edn. The John Hopkins University Press, Baltimore and London (1989) 